\title{
Abordaje transtorácico para la hidatidosis pulmonar derecha y hepática concomitante*
}

\author{
Drs. JAIME JANS B. ${ }^{1,2}$, MARÍA J. TALMA R. ${ }^{2}$, MARIO ALMONACID R. ${ }^{2}$, MAURICIO HERRERA H..$^{1,2}$ \\ Servicio de Cirugía, Hospital Base de Osorno. \\ 2 Campo Clínico Osorno, Facultad de Medicina, Universidad Austral de Chile. \\ Osorno, Chile.
}

Abstract

\section{Transthoracic approach for concurrent right lung and liver hydatid cysts}

Introduction: Pulmonary and hepatic hydatid disease occurs concomitantly in 4 to $33 \%$ of cases. When the condition is localized in the right lung and liver dome surgical resolution in one time is possible. Objective: To describe the transthoracic-transfrenic approach and review the available literature. Material and Methods: We report two cases operated at our institution through a right posterolateral thoracotomy and frenotomy in one stage, with uneventful postoperative evolution. A systematic review of the literature was conducted in the PubMed database period 1990-2011. Five studies met the inclusion criteria. Conclusions: Transthoracic-transfrenic approach is feasible and safe, allowing the surgical resolution in one time through a single incision, without another anesthetic procedure or subsequent laparotomy, with low morbidity and mortality.

Key words: Pulmonary echinoccocosis, hepatic echinoccocosis and transthoracic approach.

\section{Resumen}

Introducción: La hidatidosis pulmonar y hepática concomitante se presenta en el 4 a 33\% de los casos. Cuando la afección está localizada en el pulmón derecho y el domo hepático la resolución quirúrgica en un tiempo es posible. Objetivo: Describir el abordaje transtorácico-transfrénico y revisar la literatura disponible. Material y Métodos: Se presentan dos casos clínicos resueltos quirúrgicamente en nuestro centro a través de toracotomía posterolateral derecha y frenotomía en un tiempo, con evolución satisfactoria sin complicaciones. Se realizó una revisión sistemática de la literatura en la base de datos PubMed, período 1990-2011. Cinco estudios cumplieron los criterios de inclusión. Conclusiones: El abordaje transtorácico-transfrénico es factible y seguro, permitiendo la resolución en un tiempo quirúrgico a través de una sola incisión, sin necesidad de otro evento anestésico ni de una laparotomía posterior, con baja morbilidad y mortalidad.

Palabras clave: Hidatidosis pulmonar, hidatidosis hepática, abordaje transtorácico.

*Recibido el 3 de septiembre de 2012 y aceptado para publicación el 14 de octubre de 2012.

Los autores declaran no tener conflictos de interés.

Correspondencia: Dr. Jaime Jans B.

Av. Guillermo Bühler 1712, Osorno, Chile.

jaimejans@gmail.com 


\section{Introducción}

La ubicación pulmonar es el $2^{\circ}$ lugar más frecuente de presentación de la hidatidosis en adultos con una incidencia del 30\% aproximadamente ${ }^{1-3}$. La asociación con hidatidosis hepática concomitante se ha reportado en $5-33 \%{ }^{4-7}$, pero se han descrito cifras de hasta $60 \%{ }^{8}$. El tratamiento, al igual que en las formas aisladas es quirúrgico. La hidatidosis pulmonar no tratada puede llevar a rotura de los quistes al árbol bronquial o pleura y causar anafilaxia y aspiración. Los quistes infectados pueden evolucionar a abscesos pulmonares, empiema, fístula broncopleural, bronquiectasias y/o atelectasias ${ }^{9}$. Los quistes hidatídicos hepáticos pueden infectarse y formar abscesos o romperse a la cavidad pleural, peritoneal o árbol biliar 5 .

El tratamiento clásico de la hidatidosis pulmonar y hepática concomitante ha sido el abordaje secuencial en dos tiempos con toracotomía y laparotomía. La resolución quirúrgica transtorácica en un tiempo se ha descrito en forma exitosa con baja morbimortalidad $^{10,11}$.

El objetivo de esta comunicación es discutir los beneficios del abordaje transtorácico para la hidatidosis pulmonar derecha y hepática concomitante a propósito de 2 casos con resultados satisfactorios, describir la técnica quirúrgica y revisar la literatura.

\section{Casos clínicos}

\section{Caso 1}

Paciente de sexo masculino de 35 años de edad,

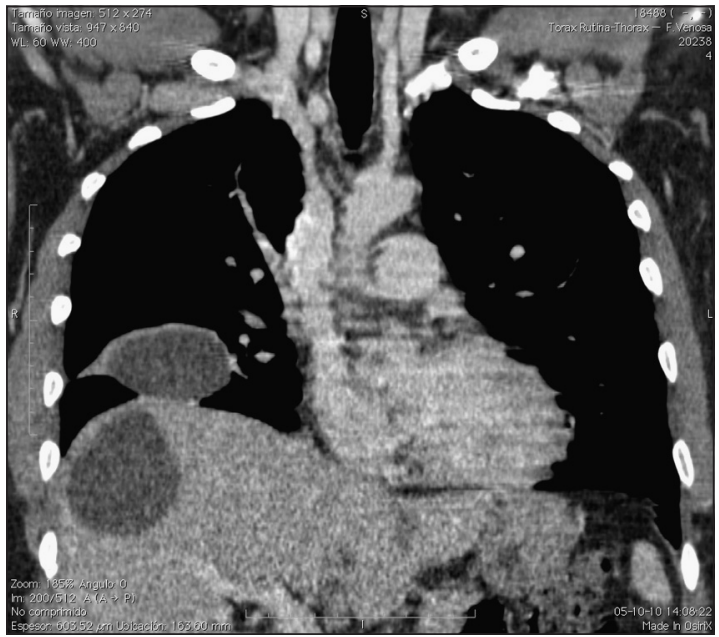

Figura 1. Caso $\mathrm{n}^{\mathrm{o}} 1$. Corte coronal de TC de Tórax. Se reconoce una lesión quística en lóbulo pulmonar inferior derecho y otra en el domo hepático. sin antecedentes mórbidos ni quirúrgicos. Consulta en el Servicio de Urgencia del Hospital Base de Osorno por cuadro de 10 días de evolución caracterizado por tos y expectoración purulenta y hemoptoica. Evoluciona con compromiso del estado general, disnea y dolor tipo puntada en hemitórax derecho inferior que se irradia a dorso con tope inspiratorio asociado a sensación febril y mialgias. Al examen pulmonar se constata murmullo pulmonar disminuido, con crépitos basales y broncofonía. Se realiza radiografía de tórax que muestra condensación basal derecha interpretada como neumonía y una imagen nodular ipsilateral. El laboratorio de ingreso presentaba PCR de $291 \mathrm{mg} / \mathrm{dl}$ y leucocitosis de $26.000 / \mathrm{mm}^{3}$. Se inicia tratamiento antibiótico empírico con Ceftriaxiona $1 \mathrm{gr} \mathrm{c} / 12 \mathrm{~h}$ y se complementa estudio con tomografía computada (TC) de tórax (Figuras 1 y 2) que muestra dos lesiones quísticas en lóbulo pulmonar inferior derecho, la mayor de ellas de $7 \mathrm{~cm}$, con características compatibles de quiste hidatídico complicado. Además se evidencia una lesión quística a nivel de la cúpula hepática de $8 \mathrm{~cm}$, estudiada complementariamente con ecografía abdominal que informa un quiste complejo en lóbulo hepático derecho, compatible con un quiste hidatídico. Una vez compensado el cuadro séptico, es operado a través de toracotomía posterolateral derecha y frenotomía radial sobre la porción tendinosa, realizando periquistectomías pulmonares parciales con cierre de comunicaciones bronquiales más capitonaje y periquistectomía total hepática sin evidenciar comunicaciones al árbol biliar. El paciente es dado de alta al $5^{\circ}$ día post operatorio en buenas condiciones.

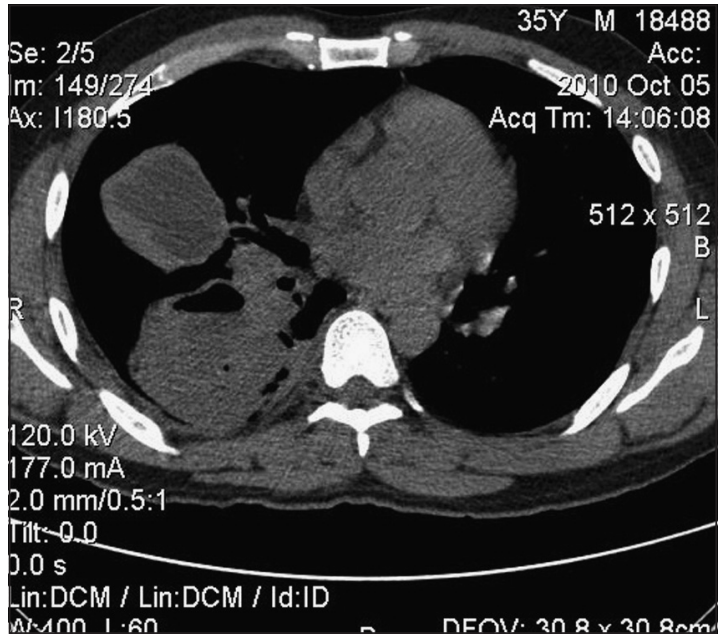

Figura 2. Caso $\mathrm{n}^{\mathrm{o}} 1$. Corte transversal de TC de Tórax. Se identifican 2 lesiones quísticas en pulmón derecho, una de ellas con nivel hidroaéreo y signos inflamatorios. 


\section{Caso 2}

Paciente de sexo femenino de 54 años de edad, con antecedente de cirugía de hidatidosis en lóbulo pulmonar inferior izquierdo y lóbulo hepático derecho, en 2 tiempos el año 2004. Consulta en Consultorio de origen por dolor torácico derecho de varias semanas de evolución, asociado a tos y expectoración hemoptoica. Se estudia ambulatoriamente con $\mathrm{Rx}$ de tórax que muestra imagen quística de gran tamaño en pulmón derecho. La serología para hidatidosis (test de ELISA IgG) resulta positiva. La tomografía computada (TC) de tórax (Figura 3) muestra 2 lesiones quísticas en lóbulo inferior de pulmón derecho, de 9 y $5 \mathrm{~cm}$ y una lesión quística multiloculada en la cúpula hepática, de $7 \mathrm{~cm}$, compatible con un quiste hidatídico en tránsito. Ingresa en forma electiva para resolución quirúrgica. Es abordada a través de toracotomía posterolateral derecha y frenotomía radial sobre la porción tendinosa, realizando periquistectomías parciales pulmonares más capitonaje y periquistectomía parcial hepática, dejando una placa de $2 \mathrm{~cm}$ de diámetro aproximadamente en el fondo de la cavidad, sin evidenciar comunicaciones al árbol biliar. La paciente es dada de alta a los 7 días post operatorios sin complicaciones.

En ambos pacientes utilizamos Albendazol postoperatorio como quimioprofilaxis, en dosis de $10 \mathrm{mg} /$ $\mathrm{kg} / \mathrm{día}$, por 3 ciclos de 21 días con 7 días de intervalo. El estudio anatomopatológico diferido confirmó en ambos pacientes quistes hidatídicos pulmonares y hepáticos.

\section{Revisión de la literatura}

Se realizó una revisión sistemática de la literatura en la base de datos PubMed, período 1990 - 2011, utilizando los términos Mesh: "Echinococcosis, Pulmonary" AND Echinoccocosis, Hepatic". Los límites de búsqueda fueron estudios en humanos, adultos e idioma inglés. Se seleccionó estudios en base a los siguientes criterios de inclusión: 1) publicado en revistas con disponibilidad de texto completo y 2) que reportaran los resultados de abordaje transtorácico-transfrénico de la hidatidosis pulmonar y hepática concomitante. Comentamos los estudios y sus resultados.

\section{Discusión}

La hidatidosis pulmonar y hepática ha sido convencionalmente tratada en 2 tiempos con toracotomía posterolateral y laparotomía diferida. También se ha descrito el abordaje en un tiempo a través de tóraco-laparotomía ${ }^{5,12}$. El abordaje transtorácicotransfrénico es una alternativa menos invasiva, que permite la resolución en un procedimiento quirúr-

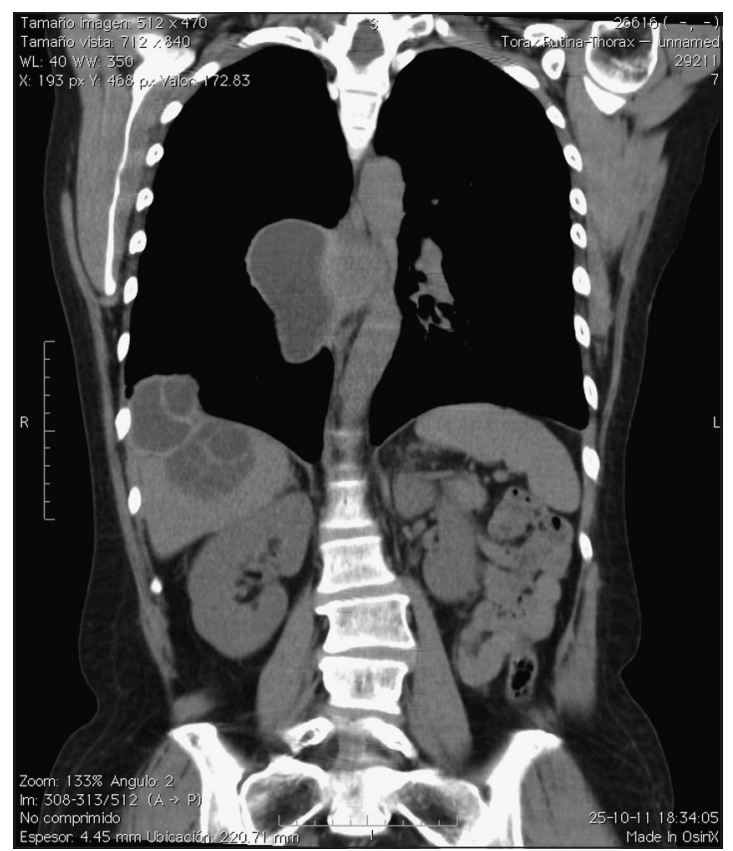

Figura 3. Caso $\mathrm{n}^{\mathrm{o}}$ 2. Corte coronal de TC de Tórax. Se reconoce una lesión quística en la región posteromedial del lóbulo pulmonar inferior derecho y otra lesión quística multiloculada en tránsito en el domo hepático.

gico y anestésico, cuando los quistes hepáticos se ubican en el domo. La intervención se lleva a cabo con el paciente bajo anestesia general con intubación pulmonar selectiva, en decúbito lateral izquierdo. A través de una toracotomía posterolateral derecha en el $6^{\circ}$ o $7^{\circ}$ espacio intercostal se accede a la cavidad pleural liberando las adherencias pleuro-pulmonares y se protege con compresas embebidas en solución fisiológica. Abordamos primero la lesión pulmonar. El quiste es puncionado y evacuado con un aspirador grueso. La parte expuesta más prominente del quiste se abre, se extraen las membranas hidatídicas y se reseca la pared libre de la lesión (periquistectomía parcial). La cavidad es lavada con solución fisiológica y mediante prueba neumática se buscan y suturan las comunicaciones bronquiales con material reabsorbible. Obliteramos la cavidad con suturas concéntricas de material reabsorbible, separadas a 1,5-2,0 $\mathrm{cm}$ desde lo más profundo (capitonaje).

Posteriormente, abordamos el quiste hepático. El diafragma es escindido radialmente en su porción tendinosa. Se liberan las adherencias hepato-frénicas y se protege el espacio subfrénico con compresas embebidas en solución fisiológica. El quiste es puncionado y aspirado. Se remueven las membranas hidatídicas y se realiza la resección del quiste en el margen del tejido hepático sano (periquistectomía 
Tabla 1. Resultados del abordaje transtorácico de la hidatidosis pulmonar y hepática concomitante

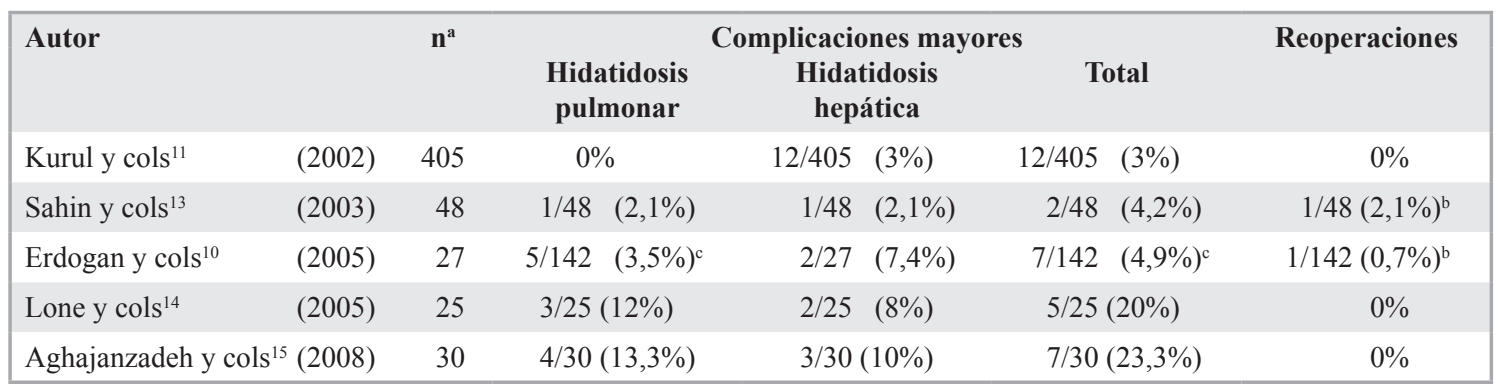

${ }^{a}$ Número de pacientes con hidatidosis pulmonar y hepática concomitante. ${ }^{b}$ Reexploración torácica por hemotórax. ${ }^{\mathrm{C}}$ Porcentaje reportado sobre el total de pacientes de la serie $(n=142)$.

total), ligando vasos y conductos biliares. De no ser posible la periquistectomía total por proximidad a estructuras vasculares o biliares centrales, recomendamos la periquistectomía parcial, dejando una placa de periquística en el fondo de la cavidad, suturando toda comunicación biliar. Obliteramos la cavidad hepática con sutura discontinua de material reabsorbible. Dejamos un drenaje al espacio subfrénico y realizamos la frenorrafia con sutura irreabsorbible. Dejamos 2 pleurostomías, apical anterior y basal posterior. El drenaje abdominal se retira cuando el débito es $<30 \mathrm{cc} /$ día y no hay tinte bilioso; y las pleurostomías, en forma secuencial, cuando la $\mathrm{Rx}$ de tórax certifica expansión pulmonar, no hay fuga aérea y los débitos son $<100$ cc/día.

Con los términos y límites de búsqueda descritos se encontró 107 artículos, de los cuales 5 cumplieron los criterios de inclusión. Tabla 1.

Kurul y cols ${ }^{11}$, reportaron una morbilidad del 12/405 (3\%) pacientes: drenaje biliar excesivo $=9$ y fistula broncobiliar $=3$; sin necesidad de reoperación y sin mortalidad perioperatoria. Luego de un seguimiento de 2 a 10 años, la recurrencia pulmonar fue $1,5 \%$, sin recurrencia hepática. Sahin y cols ${ }^{13}$, reportaron complicaciones mayores en $2 / 48(4,2 \%)$ pacientes: hemorragia con necesidad de reexploración $=1$ y fistula biliocutánea $=1 ;$ sin mortalidad perioperatoria y con recurrencias pulmonares y hepáticas de 2,1\% y 6,2\% respectivamente. Erdogan y $\mathrm{cols}^{10}$, en una serie de 142 pacientes con hidatidosis pulmonar, realizaron el abordaje transtorácicotransfrénico en 27 pacientes con hidatidosis hepática concomitante localizada en el domo. Si bien no se detalla la morbilidad del grupo en particular, reportan complicaciones mayores en 7/142 (4,9\%) pacientes (empiema $=3$, hemorragia $=1$, fistula bronco pleural $=1$ y drenaje biliar excesivo $=2$ (definido como $>7$ días). No hubo mortalidad perioperatoria. La recurrencia pulmonar fue de $5,6 \%$ y la hepática de $0 \%$, con un seguimiento de
$28 \pm 6,8$ meses. Lone y cols ${ }^{14}$, realizaron el abordaje transtorácico-transfrénico a través de toracotomía anterior en 25 pacientes, con baja reserva pulmonar y quistes no complicados, menores de $10 \mathrm{~cm}$. El tiempo operatorio promedio fue $75 \mathrm{~min}$ (rango de 70 a $110 \mathrm{~min}$ ) y el promedio de hospitalización fue de 5,2 días. Tres (12\%) pacientes presentaron fuga aérea $>10$ días y $2 / 25(8 \%)$ drenajes biliares prolongados. No hubo reoperaciones ni mortalidad. Por último, Aghajanzadeh y cols ${ }^{15}$, reportaron una morbilidad de $7 / 30(23,3 \%)$ pacientes: empiema $=2$, fístula broncopleural $=1$, drenaje biliar excesivo $=3$ y hemorragia $=1$. El rango de hospitalización fue de 6 a 12 días. No hubo mortalidad en la serie. Hubo $2 / 30(6,6 \%)$ recurrencias hepáticas luego de un seguimiento de 12 a 36 meses.

Los autores concluyen que el abordaje es factible, seguro y efectivo, limitando al trauma a un espacio intercostal, debiendo ser considerado con el objeto de prevenir una segunda intervención. La morbilidad y mortalidad es concordante con las cifras reportadas para el tratamiento quirúrgico de la hidatidosis pulmonar (1,4-19,1\% y $0,6-4,2 \%$, respectivamente) ${ }^{16-18}$ y para la hidatidosis hepática (6-47\% y $0-3 \%$, respectivamente) ${ }^{19}$.

En nuestro centro hemos realizado el abordaje transtorácico-transfrénico en 2 pacientes, con resultados satisfactorios, apoyando que es una técnica factible y segura en el tratamiento de la hidatidosis pulmonar derecha y hepática concomitante, localizada a nivel del domo, permitiendo la resolución en un tiempo quirúrgico a través de una sola incisión, sin necesidad de otro evento anestésico ni de una laparotomía posterior, con baja morbilidad y mortalidad.

\section{Referencias}

1. Dogan R, Yuksel M, Cetin G, Suzer K, Alp M, Kaya $\mathrm{S}$, et al. Surgical treatment of hydatid cysts of the lung: report on 1055 patients. Thorax 1989;44:192-9. 
2. Burgos R, Varela A, Castedo E, Roda J, Montero CG, Serrano S, et al. Pulmonary hydatidosis: surgical treatment and follow-up of 240 cases. Eur J Cardiothorac Surg. 1999;16:628-35.

3. Ramos G, Orduna A, García-Yuste M. Hydatid cyst of the lung: diagnosis and Treatment. World J Surg. 2001;25:46-57.

4. Rami-Porta R, Aisconde JG, Bravo-Bravo JL, AlixTrueba A, Serrano- Muñoz F. Treatment of synchronous pulmonary and hepatic hydatid cysts. J Thorac Cardiovasc Surg. 1986;92:314-5.

5. Eren N, Ozgen G. Simultaneous operation for right pulmonary and liver echinococcosis. Scand J Thorac Cardiovasc Surg. 1990;24:131-4.

6. Rojas M, González L, Sagredo R. Hidatidosis Pulmonar en el Hospital de Coquimbo. Rev Chil Cir. 2002;54:661-4.

7. Jans J, Bórquez P, Marambio A, Manoli P, Hollstein A, Zarges P. Resultados del tratamiento de la hidatidosis pulmonar complicada y no complicada. Rev Chil Cir. 2012;64:346-51.

8. Vera G, Venturelli F, Ramírez J, Venturelli A. Hidatidosis Humana. Cuad. Cir. 2003;17:88-94.

9. Morris DL, Richards KS. Textbook of Hydatid Disease: Current Medical and Surgical management (1st edn). Butterworth-Heinemann: London, 1992;25-30.

10. Erdogan A, Ayten A, Kabukcu H, Demircan A. One-stage transthoracic operation for the treatment of right lung and liver hydatid cysts. World J Surg. 2005;29:1680-6.

11. Kurul IC, Topcu S, Altinok T, Yazici U, Tastepe I, Kaya
$\mathrm{S}$, et al. One-stage operation for hydatid disease of lung and liver: principles of treatment. J Thorac Cardiovasc Surg. 2002;124:1212-5.

12. Yalcinkaya I, Er M, Ozbay B, Ugras S. Surgical treatment of hydatid cyst of the lung: review of 30 cases. Eur Respir J. 1999;13:441-4.

13. Sahin E, Enön S, Cangir AK, Kutlay H, Kavukçu S, Akay $\mathrm{H}$ et al. Single-stage transthoracic approach for right lung and liver hydatid disease. J Thorac Cardiovasc Surg. 2003;126:769-73.

14. Lone GN, Bhat MA, Ali N, Ahangar AG, Dar AM, Sana I. Minimally invasive transthoracotomy-transphrenotomy for concurrent hepatic and pulmonary hydatid disease. Br J Surg. 2005;92:729-33.

15. Aghajanzadeh M, Safarpoor F, Amani H, Alavi A. Onestage procedure for lung and liver hydatid cysts. Asian Cardiovasc Thorac Ann. 2008;16:392-5.

16. Burgos R, Varela A, Castedo E, Roda J, Montero CG, Serrano S, et al. Pulmonary hydatidosis: surgical treatment and follow-up of 240 cases. Eur J Cardiothorac Surg. 1999;16:628-35.

17. Kuzucu A, Soysal O, Ozgel M, Yologlu S. Complicated hydatid cyst of the lung: clinical and therapeutic issues. Ann Thorac Surg. 2004;77:1200-4.

18. Arıbas, OK, Kanat F, Türk E, Kalaycı MU. Comparison between pulmonary and hepatopulmonary hydatidosis. Eur J Cardiothorac Surg. 2002;21:489-96.

19. Magistrelli P, Masetti R, Coppola R. Surgical treatment of disease of liver. Arch Surg. 1991;126:518-23. 